\title{
Additive manufacturing method of prototyping novel mm-wave and THz sources
}

\author{
A. R. Phipps, A. J. MacLachlan, C. W. Robertson, A. W. Cross, and A. D. R. Phelps \\ Department of Physics, SUPA, University of Strathclyde, Glasgow, G4 ONG, Scotland, UK \\ a.d.r.phelps@strath.ac.uk
}

\begin{abstract}
To rapidly prototype novel mm-wave and $\mathrm{THz}$ sources there is a requirement to create intricate structures to produce and radiate electromagnetic fields. The motivation for this work is to create improved electron-beam-driven, vacuum electronic mm-wave and sub-THz sources by exploiting dispersion engineering. Although such structures can be manufactured by other techniques, additive manufacturing has proven to be quick, reliable and cost-effective. This research is allowing the prototyping of novel mm-wave and sub- THz coherent sources.
\end{abstract}

Keywords - additive manufacturing; mm-wave sources; periodic structures; $3 D$ printing.

\section{INTRODUCTION}

Additive manufacture [1] also known as "3D printing" is becoming more widely available as a method for manufacturing rapidly and cost-effectively small quantities of intricate structures. Such structures are of great interest because the application of smart dispersion engineering and transformational electromagnetics is having a positive impact on research leading to improved high-power coherent sources [2-6]. Two dimensional periodic surface lattices (PSLs) [7]. can be created by manufacturing shallow periodic perturbations on the inner surface of a hollow electrically conducting cylinder. Such 2D PSLs can be used for several applications exploiting different physical phenomena, [2-10]. A dispersion relation describing the coupling of the volume and surface fields inside a 2D PSL of cylindrical topology was derived by Konoplev et al. [9]. Under certain conditions, when driven by a suitable electron beam this structure can support a Cherenkov instability that provides a coherent source of electromagnetic radiation. These cylindrical 2D PSLs show potential for use in electron-beam-driven high power, coherent sources [7,9-11].

The cylindrical PSL structures need to be compatible with vacuum conditions and the use of energetic electron beams, while also providing the required boundary conditions for the electromagnetic fields. Manufacturing the cylindrical PSLs out of a suitable metal usually provides a good vacuum envelope and the electrical conductivity allows electrical charges impacting on the surfaces to be conducted away. A reasonably high thermal conductivity coefficient is another desirable property for PSLs intended for use in high power sources.
In our earlier years of research on high power mm-wave sources the technique employed to manufacture the cylindrical PSLs was to mill sinusoidal perturbations on the outer wall of an aluminium former and then to electrodeposit copper directly onto the surface. The aluminium was then dissolved in a strong alkaline solution, leaving the copper PSL with the perturbations on the inner surface.

\section{RESULTS}

The 3D printing technology used has reasonably high resolution, producing objects that have resolutions on the \pm 16 micron scale. The dimensional errors achievable with $3 \mathrm{D}$ printing are projected to decrease over time as the technology develops. Fig. 1(a) shows a photograph of a cylindrical silver-chromium alloy $2 \mathrm{D}$ periodic surface lattice, cast using a primary mandrel manufactured by $3 \mathrm{D}$ printing. The perturbations on the inner surface of the metallic cylinder are sinusoidal, whereas the virtual schematic of the mandrel seen in Fig. 1(b) just to indicate the dimensions has rectangular perturbations.

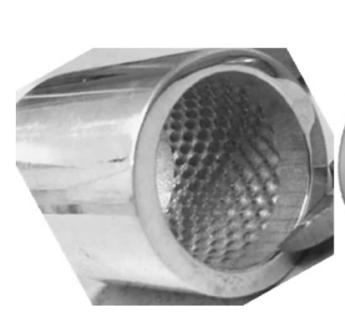

(a) (b)

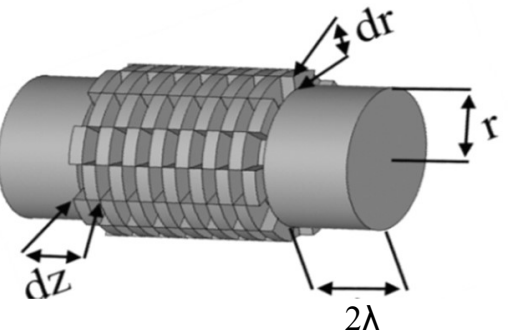

Fig. 1. (a) Cherenkov source cavity consisting of a cylindrical silverchromium alloy 2D periodic surface lattice (b) Cylindrical CAD virtual mandrel that when constructed using additive manufacturing provided the mold for casting the cylindrical 2D PSL.

This cylindrical metallic 2D PSL is designed for a Cherenkov source to operate in the $\mathrm{W}$-band $(75-110 \mathrm{GHz})$ and the values of the parameters shown in Fig. 1 are $r=10.0 \mathrm{~mm}$, $\mathrm{dz}=3.0 \mathrm{~mm}, \mathrm{dr}=0.40 \mathrm{~mm}$ and $2 \mathrm{r} / \lambda \sim 7$.

To measure the properties of the lattice the electromagnetic wave-beam was formed using the set up shown in fig. 2 which is capable of converting the fundamental $\mathrm{TE}_{1,1}$ mode of a cylindrical waveguide into either the $\mathrm{TM}_{0,1}$ mode of a cylindrical waveguide, or the TEM mode of a coaxial waveguide. The wave-beam was then launched via either a 
Author Accepted Manuscript: Paper presented at IEEE 9th UK-Europe-China Workshop on Millimetre Waves and Terahertz Technologies (UCMMT), 5-7 September 2016, Qingdao, China. Accepted paper (3 pages) published online by IEEE Xplore, 09 March 2017.

co-axial line termination plane launcher (such that the lattice is irradiated at all possible angles simultaneously) or via a directly irradiating structure with the $\mathrm{TM}_{0,1}$ wave-beam (oblique irradiation of the lattice at a small grazing angle).

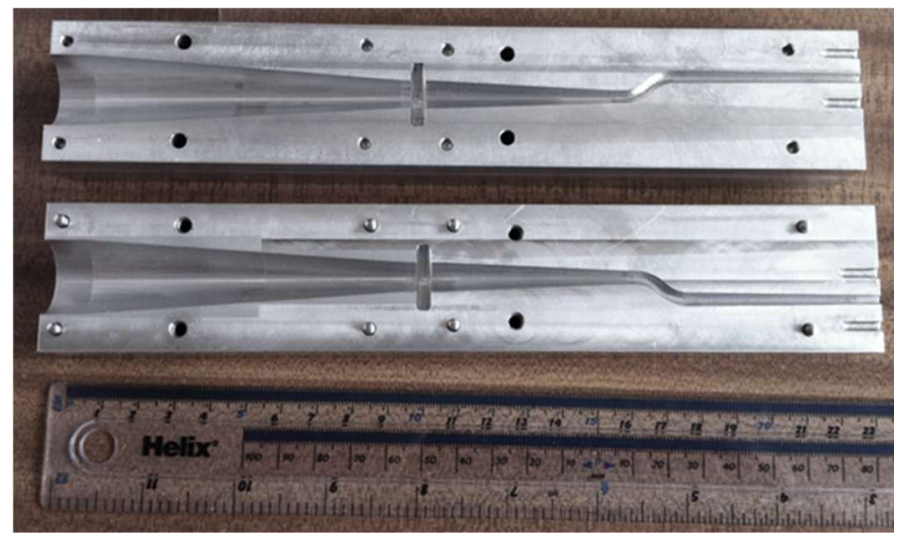

Fig. 2. Photograph of the experimental set-up designed to measure 2D PSLs as shown above.

The measurements of the PSLs were conducted in the far field zone ( $27 \mathrm{~cm}$ from the output aperture) and millimetrewave absorbent material was used to reduce parasitic reflections. A millimetre-wave Anritsu Vector Network Analyser was used for the measurements.

Fig. 3 shows measurements, made using a VNA, of the electromagnetic properties of this W-band cylindrical PSL and for comparison two simulations, made using CST Microwave Studio using the co-axial launcher scattering a near cut-off wave on the lattice. The results show the potential of this structure for creating a novel coherent source when excited by a suitable electron beam.

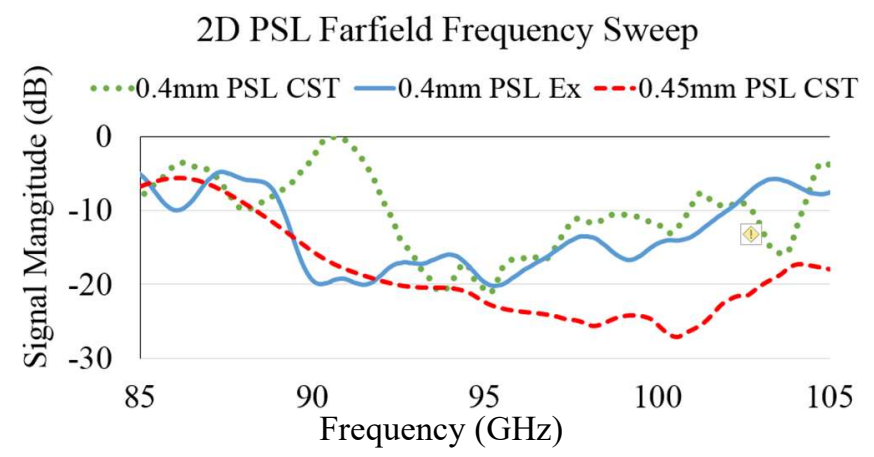

Fig. 3. On-axis frequency sweep for the W-band 2D PSL. Experimental measurements shown as the solid line. Simulations of the structure shown for comparison, one with a perturbation amplitude of $0.4 \mathrm{~mm}$ (dotted line) and one with $0.45 \mathrm{~mm}$ (dashed line).

A structure with a high amplitude of perturbations $0.8 \mathrm{~mm}$ was measured to investigate the high contrast case, as its peak-to-peak corrugation of $1.6 \mathrm{~mm}$ (from top to bottom of the grating) is larger than a quarter of the operating wavelength. The co-axial launcher was substituted with the direct launcher to excite the structure with a propagating mode with the millimetre wave transmission measured using the Vector Network Analyser as shown in figure 4.

$$
\text { Frequency }(\mathrm{GHz})
$$

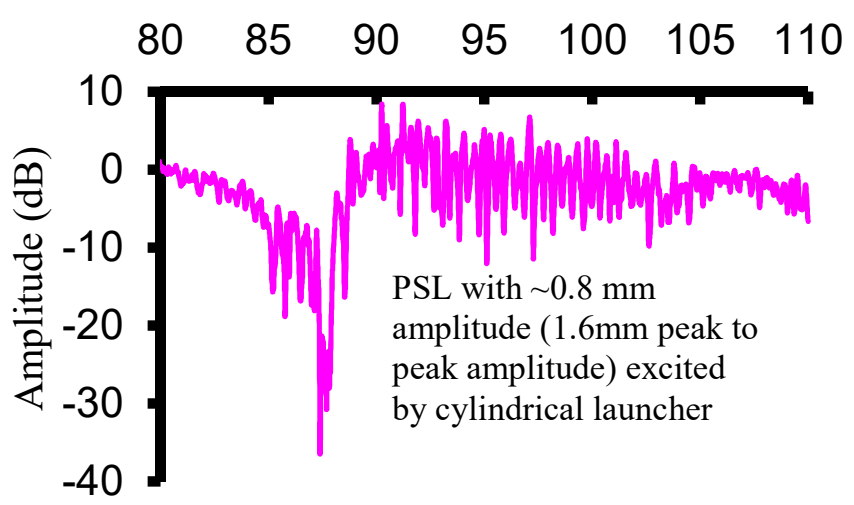

Fig. 4. Results of measurements of the electromagnetic signal transmission versus frequency (2D PSL spectral response) through a high contrast $2 \mathrm{D}$ PSL. The structure was irradiated by an oblique (near-grazing) incident wave-beam formed by the $\mathrm{TM}_{01}$ mode of the cylindrical waveguide.

The cavity eigenmode formation of a high contrast 2D PSL was modelled using CST Microwave studio and is shown in figure 5 .
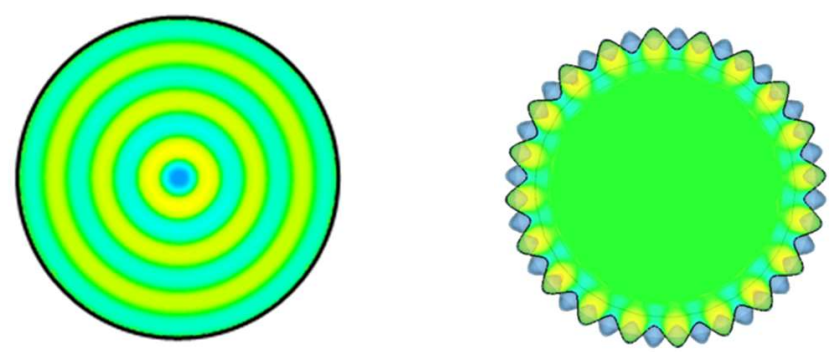

Volume field TM 0,7 couples to surface wave HE20.1

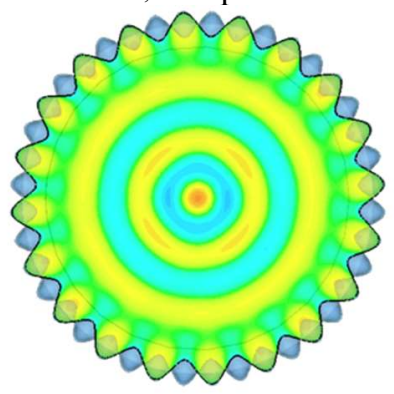

Cavity eigenmode

Fig. 5. CST Microwave Studio modelling showing the coupling of a volume wave and a surface wave on a high contrast 2D PSL structure and cavity eigenmode formation.

Comparing figures 3, 4 and 5 we see that the position of the minima observed for the high contrast lattice as compared to the lower contrast $0.4 \mathrm{~mm}$ structure is associated with the eigenmode having 7 radial variations (formed with the partial $\mathrm{TM}_{07}$ mode) and having downshifted minima around $87 \mathrm{GHz}$. 
Author Accepted Manuscript: Paper presented at IEEE 9th UK-Europe-China Workshop on Millimetre Waves and Terahertz Technologies (UCMMT), 5-7 September 2016, Qingdao, China. Accepted paper (3 pages) published online by IEEE Xplore, 09 March 2017.

To excite such eigenmodes an annular electron beam [12] should be positioned at the optimum radius.

\section{CONCLUSIONS}

Cylindrical 2D PSL structures have been successfully protoyped using additive manufacturing to create the primary mold. This primary mold was then used to cast successfully the metallic cylindrical 2D PSL which forms the interaction cavity for a novel mm-wave source. This additive manufacturing method that has already been successfully used in lower frequency microwave sources [13] has been shown in our present work to be capable of prototyping dispersive structures and complex components for novel mmwave and sub-THz sources and may have the potential to be extended to manufacture structures relevant to even higher frequencies [14].

At present the structural resolution attainable using additive manufacturing techniques makes the achievement of structures capable of generating frequencies in the true $\mathrm{THz}$ frequency range challenging. The further development of additive manufacturing technology to facilitate the reliable production of even finer scale structures would allow similar sources to these novel mm-wave and sub- $\mathrm{THz}$ sources to be manufactured for operation at $\mathrm{THz}$ frequencies.

\section{ACKNOWLEDGMENT}

Alan R. Phipps and Amy J. MacLachlan thank the EPSRC for supporting their postgraduate studentships. D. Barclay provided valuable technical assistance. This work was partly funded by a Leverhulme Trust International Grant Network on "Advanced research on generation of $\mathrm{THz}$ and X-ray radiation". The authors would also like to thank EOARD for partial support of this work.

\section{REFERENCES}

[1] I. Gibson, D. Rosen and B. Stucker, "Additive Manufacturing Technologies: 3D Printing, Rapid Prototyping, and Direct Digital Manufacturing", 2nd Edition, Springer, New York, USA, 2015.

[2] N. S. Ginzburg, et al., "Theory of free-electron maser with twodimensional feedback driven by an annular electron beam", J. Appl. Phys., vol. 92, pp. 1619-1629, Aug. 2002.

[3] N. S. Ginzburg, et al., "The use of a hybrid resonator consisting of one dimensional and two-dimensional Bragg reflectors for generation of spatially coherent radiation in a co-axial free-electron laser", Phys. Plasmas, vol. 9, pp. 2798-2802, June 2002

[4] A. W. Cross, et al., "Experimental and theoretical study of 2D Bragg structures for a coaxial free electron maser", Nuclear Instruments and Methods in Physics Research A, vol. 475, pp. 164-172, Dec. 2001.

[5] A. W. Cross, et al., "Experimental studies of two dimensional coaxial Bragg structures for a high-power free-electron maser", Appl. Phys. Lett. vol. 80 , pp. $1517-1519$, March 2002.

[6] I. V. Konoplev, et al., "Experimental and theoretical studies of a coaxial free-electron maser based on two-dimensional distributed feedback", Phys. Rev. E, vol. 76, 056406, Nov. 2007.

[7] I. V. Konoplev, et al., "Surface wave Cherenkov maser based on a periodic lattice", Appl. Phys. Lett., vol. 96, 261101, June 2010.

[8] A. W. Cross, et al., "Studies of surface two-dimensional photonic bandgap structures”, J. Appl. Phys., vol. 93, pp. 2208-2218, Feb. 2003.
[9] I. V. Konoplev, et al., "Cylindrical, periodic surface lattice - Theory, dispersion analysis and experiment", Appl. Phys. Lett., vol. 101, 121111, Sept. 2012.

[10] I. V. Konoplev, et al., "Cylindrical periodic surface lattice as a metadielectric: concept of a surface-field Cherenkov source of coherent radiation", Phys. Rev. A, vol. 84, 013826, July 2011.

[11] I. V. Konoplev, et al., "Surface field excitation by an obliquely incident wave", Appl. Phys. Lett., vol. 102, 141106, April 2013.

[12] I. V. Konoplev, et al., "high current oversized annular electron beam formation for high-power microwave research", Appl. Phys. Lett., vol. 89, 171503, Oct. 2006

[13] D. M. French, and D. Shiffler, "High power microwave source with a three dimensional printed metamaterial slow-wave structure", Rev. Sci. Instrum., vol. 87, no. 5, 053308, May 2016.

[14] T. Zhao, et al., "Enhanced diffraction radiation from two dimensional periodical structure", The European Physical Journal D, vol. 68, no. 10, pp. 1-5, Oct. 2014. 